\title{
Alban Bensa, Après Lévi-Strauss. Pour une anthropologie à taille humaine
}

Paris, Éditions Textuel, coll. « Conversations pour demain », 2010, 128 p.

Sophie Blanchy

\section{OpenEdition}

\section{Journals}

Édition électronique

URL : http://journals.openedition.org/assr/23447

DOI : $10.4000 /$ assr.23447

ISSN : $1777-5825$

Éditeur

Éditions de l'EHESS

Édition imprimée

Date de publication : 31 décembre 2011

Pagination : 112

ISBN : 9782713223273

ISSN : 0335-5985

\section{Référence électronique}

Sophie Blanchy, «Alban Bensa, Après Lévi-Strauss. Pour une anthropologie à taille humaine », Archives de sciences sociales des religions [En ligne], 156 I octobre-décembre 2011, document 156-18, mis en ligne le 14 février 2012, consulté le 21 septembre 2020. URL : http://journals.openedition.org/assr/ 23447 ; DOI : https://doi.org/10.4000/assr.23447

Ce document a été généré automatiquement le 21 septembre 2020

(C) Archives de sciences sociales des religions 


\section{Alban Bensa, Après Lévi-Strauss. Pour une anthropologie à taille humaine}

Paris, Éditions Textuel, coll. « Conversations pour demain », 2010, 128 p.

\section{Sophie Blanchy}

\section{RÉFÉRENCE}

Alban BENSA, Après Lévi-Strauss. Pour une anthropologie à taille humaine, Paris,

Éditions Textuel, coll. «Conversations pour demain », 2010, 128 p.

1 Ce petit livre au titre choc (la disparition de la figure tutélaire de l'anthropologie libèrerait-elle l'expression critique?) constitue une bonne introduction au débat contemporain entre deux grands courants de l'anthropologie : l'un visant la montée en généralité (structuraliste ou fonctionnaliste) et l'autre donnant toute son attention au contexte, au contingent et à l'historique. Les entretiens publiés dans cette collection font le tour d'une question d'actualité en sciences sociales à l'adresse d'un public averti. Celui-ci propose une synthèse vivante de la pensée d'Alban Bensa, qui s'inscrit dans le "retour au réel » opéré après la période structuraliste de la discipline, déjà mise à distance dans les dernières décennies, et dénonce le culturalisme. Le dialogue est découpé en trois parties : Après Lévi-Strauss, revenir au réel; Totem et tabou : les fables d'une discipline; L'anthropologue et le politique. L'auteur y discute quelques grands concepts de la discipline et convoque une série d'auteurs avec lesquels il se reconnaît des affinités intellectuelles, tout en rappelant ses propres propositions théoriques et méthodologiques (voir notamment La fin de l'exotisme, 2006, recueil de textes publiés entre 1984 et 2003, et son avant-propos). Il défend en tout premier lieu l'idée de l'historicité des sociétés, du travail du temps qui les constitue, contre les modèles décontextualisés qui, en recherchant des lois générales, aboutissent à une séparation complète du symbolique et du social et renvoient une image d'ordre qui ne 
rend pas compte du réel. Ce que Bensa reproche notamment à la pensée de LéviStrauss, c'est d'avoir laissé de côté ce qui trouble ou qui bouleverse (par exemple, la Shoah ou l'expérience de l'inceste), privilégiant la stabilité formelle aux dépens des contenus, des idéologies, des choix politiques, des émotions. Ainsi, à la notion de règle matrimoniale, A. Bensa oppose les stratégies politiques d'alliance, mises en évidence par Pierre Bourdieu en Kabylie, par exemple, qui permettent de jouer à la fois selon et contre la règle affichée. Emmanuel Terray notait déjà en 1999 que le structuralisme de Lévi-Strauss ne permettait pas d'appréhender l'action, avec ce qu'elle implique d'incertitude; de même la violence, ses sources et ses effets dans l'histoire, font aujourd'hui l'objet du recentrement de certains travaux. Refusant donc les modèles trop généraux, Bensa opte pour une "conceptualisation basse " qui permet des recoupements à partir de descriptions fouillées, descriptions dont Jean Bazin avait fait le cœur du travail d'explicitation de l'action des autres, pour lui le seul programme de la discipline (voir la préface d'Alban Bensa à Des clous dans la Joconde, recueil de textes de Jean Bazin, publié en 2008).

2 L'enquête de terrain, sur laquelle l'auteur a publié, avec Didier Fassin, des travaux de jeunes chercheurs, n'assure un régime de scientificité adéquat aux sciences humaines que si elle est questionnée et réfléchie comme un dispositif spécifique. Elle doit avoir la forme d'une véritable interlocution, dans la proximité avec les autres que seuls assurent de longs séjours répétés. Le regard rapproché, qui implique la prise en compte de la subjectivité du chercheur, est le "moyen de ne pas céder aux facilités de la généralisation » que permettait le «regard éloigné » de Levi-Strauss. Il ne s'agit pas pour autant de remettre en cause le décalage, longtemps inculqué comme point de méthode, devant permettre l'explicitation de tout ce qui va de soi dans un univers social différent du sien. L'auteur s'est lui-même formé sur un terrain lointain par la découverte progressive des «injonctions sociales spécifiques au monde dont on fait l'expérience » (p. 38-39).

3 La lutte contre la désindexation des faits sociaux et autres « fables de la discipline » est aujourd'hui un combat d'arrière-garde : la France est le dernier champ d'une bataille engagée partout dans le monde dès le milieu des années soixante-dix. On peut se demander si l'auteur, emporté par sa conviction, ne fait pas un mauvais procès à une anthropologie qui, pour se livrer à l'exercice comparatif, se condamne à hypostasier la société, la culture et leurs règles inconscientes. Le retour à l'action, au choix des individus, oblige-t-il à rejeter toute idée d'entités collectives partagées de manière quelque peu stable? Sinon, comment identifier celles-ci sans jamais en faire des totalités surplombant les individus? On comprend cependant le souci méthodologique de rigueur qui anime l'auteur et qu'il partageait avec Jean Bazin. Ce dernier rappelait d'une part que la culture n'est ni une cause, ni même un fait, mais tout simplement « le produit d'une opération ethnographique ", et soulignait d'autre part que les conditions de la connaissance ne sont jamais totalement réunies (Bensa, 2008, citant Bazin 1996: «Interpréter ou décrire. Notes critiques sur la connaissance anthropologique » in Revel \& Wachtel, éds, Une école pour les sciences sociales. De la VI section à l'École des hautes études en sciences sociales). Au grand récit explicatif, Bensa préfère donc les histoires racontées par les gens. Elles révèlent l'usage qu'ils font de règles qui, énoncées de manière isolée, ne nous disent rien du réel à comprendre. D'ailleurs, jamais données à l'ethnologue, ces règles ne s'acquièrent qu'en apprenant à jouer au jeu social du terrain d'enquête. Face à la question du comparatisme, l'auteur est néanmoins confronté aux outils de pensée 
dénoncés. Il considère par exemple le rôle des oncles maternels dans les sociétés du Pacifique, qu'il se refuse à définir comme un «trait culturel» (p. 72), comme « une idée forte, récurrente et stable », (p. 73) et il identifie également « au fond des attitudes et des pensées, la logique segmentaire ", " plus encadrante que le pouvoir attribué aux oncles maternels » (p. 76). Mais il souligne la temporalité de ces « configurations », leur durée historique qui peut être courte, et aussi leur place dans la vie quotidienne où l'oncle maternel par exemple est à la fois une inquiétude et une ressource (p. 74). Il s'agit alors de ne comparer que les possibilités qu'ont les acteurs (le sens pratique de Bourdieu), déployées dans un éventail plus ou moins ouvert selon la position de chacun, qui leur permettent de jouer coup par coup - la justification viendra après - sans que jamais ne s'efface un certain degré de contingence. Face aux qualificatifs d'empiriste ou de nominaliste dont il a été affublé, l'auteur assume ses positions : il est de ceux qui pensent que la position de surplomb du chercheur entraîne une trop grande perte de sens, et, en s'appuyant à la fois sur Wittgenstein et sur Bazin, "qu'une bonne description vaut toutes les interprétations » (p. 86).

Dénonçant sans cesse une conception fixiste des « ethnies » et de la «culture » dont il a lui-même fait l'expérience dans le contexte postcolonial de la Nouvelle-Calédonie, il prend acte cependant avec réalisme de la remontée des sentiments d'appartenance ethnique partout dans le monde et de «l'extraordinaire efficacité contemporaine de la notion d'ethnie» (p.94), quelle qu'en soit son origine historique. L'ethnologie doit assumer sa place de spécialiste non de l'ethnie, mais des configurations historiques et sociales qui la produisent à un moment donné, non sans l'influence de l'État et de ses outils de fabrication des identités (les papiers : comme on le voit aujourd'hui à Mayotte, à propos de laquelle le terme d'ethnie apparait sous certaines plumes sociologiques, à l'encontre du sens commun local, pour définir des ensembles sociaux produits par la discrimination étatique, en particulier celui des migrants anjouannais). Pour l'auteur, le musée du quai Branly y participe en induisant, par son esthétique du lointain, une homologie identitaire entre l'ethnie et son art, aux dépens d'une histoire singulière des objets, de leur circulation et de leur créateur, dans une démarche où se lit l'effet direct de la pensée lévi-straussienne. Dans le même temps, le musée de la Porte Dorée présente l'histoire (migratoire) de certains des peuples ethnicisés au quai Branly. L'auteur réfléchit à la notion d'identité culturelle à la lumière de son expérience partagée avec les Kanaks. Il définit quel a été son double engagement : comprendre scientifiquement le monde kanak en intégrant l'histoire, soutenir politiquement son projet de dignité et d'indépendance. Dans un contexte de forte domination, dans les DOM-TOM postcoloniaux comme en métropole entre élite et population ou face aux migrants, il plaide pour une ethnologie de la proximité capable de détisser les ordres, de casser les hiérarchies, d'expliciter «les pratiques réelles des acteurs dans leur propre univers » (p.44) et d'aider au dialogue avec les autorités afin que celles-ci, au lieu de trancher unilatéralement, acceptent d'impliquer ceux que les décisions concernent. Quant aux options morales et politiques de l'ethnologue sur son terrain, à chacun de faire son choix, car toute société, loin d'être un système unique, est une confrontation de systèmes de valeurs différents et la violence y est toujours présente. Bensa a pris position quant à lui sur l'avenir politique de la Kanaky.

5 En opposition au positivisme et au scientisme qu'il récuse - et qui renaît sous de nouvelles formes - A. Bensa évoque dans cet entretien plusieurs travaux d'anthropologie, récents ou précurseurs en leur temps, fondés sur une réflexivité maximale, attentifs à l'intersubjectivité et aux situations en construction, préférant 
parler de différence que d'altérité, faisant du sujet singulier une priorité et prenant en compte son malaise d'être. Le dernier mot sera donné, parmi les nombreux auteurs cités, à Jean Bazin avec qui A. Bensa a dirigé pendant dix ans un séminaire à l'EHESS et dont la pensée exigeante et originale l'a constamment stimulé : pour approcher au plus près de la réalité sociale, il faut « prendre l'autre au sérieux » (p. 123). 